JOURNAL OF

APPLIED

CRYSTALLOGRAPHY

ISSN 1600-5767

Received 28 April 2021

Accepted 28 April 2021

Keywords: surface diffraction; Bayesian inference; Monte Carlo; oxide films; epitaxial films

\section{Robust surface structure analysis with reliable uncertainty estimation using the exchange Monte Carlo method. Corrigendum}

\author{
Kazuki Nagai, ${ }^{a}$ Masato Anada, ${ }^{a}$ Yoshinori Nakanishi-Ohno, ${ }^{\mathrm{b}, c, d}$ Masato Okada ${ }^{\mathrm{e}}$ and \\ Yusuke Wakabayashi ${ }^{\mathrm{f}}$.
}

${ }^{a}$ Graduate School of Engineering Science, Osaka University, 1-3 Machikaneyama, Toyonaka, Osaka 560-8531, Japan, ${ }^{\mathbf{b}}$ Graduate School of Arts and Sciences, The University of Tokyo, 3-8-1 Komaba, Meguro, Tokyo 153-8902, Japan, ${ }^{{ }}$Komaba Institute for Science, The University of Tokyo, 3-8-1 Komaba, Meguro, Tokyo 153-8902, Japan, ${ }^{\mathbf{d}}$ Precursory Research for Embryonic Science and Technology, Japan Science and Technology Agency, 4-1-8 Honcho, Kawaguchi, Saitama 332-0012, Japan, ${ }^{\mathbf{e} G r a d u a t e}$ School of Frontier Sciences, The University of Tokyo, 5-1-5 Kashiwanoha, Kashiwa, Chiba 277-8561, Japan, and ${ }^{\mathrm{f} G r a d u a t e}$ School of Science, Tohoku University, 6-3, Aramaki Aza-Aoba, Aoba-ku, Sendai 980-8578, Japan. *Correspondence e-mail: wakabayashi@tohoku.ac.jp

An error in the article by Nagai, Anada, Nakanishi-Ohno, Okada \& Wakabayashi [J. Appl. Cryst. (2020), 53, 387-392] is corrected.

Equation (11) in the article by Nagai et al. (2020) should read

$$
C_{\mathrm{E}}(j)=\frac{\sum_{i} \Delta\left(\mathbf{Q}_{i}\right) \Delta\left(\mathbf{Q}_{i+j}\right)}{\sum_{i}\left[\Delta\left(\mathbf{Q}_{i}\right)\right]^{2}} .
$$

The inset of Fig. 6 was calculated with the correct equation, and therefore no change is needed in the rest of the paper.

\section{References}

Nagai, K., Anada, M., Nakanishi-Ohno, Y., Okada, M. \& Wakabayashi, Y. (2020). J. Appl. Cryst. 53, 387-392.

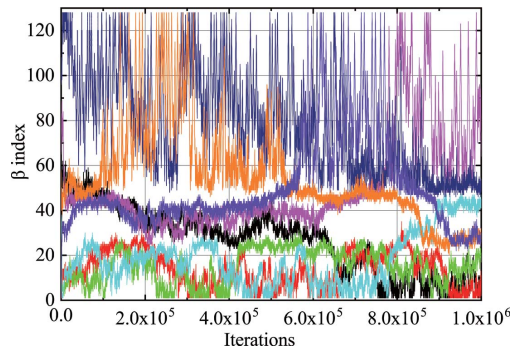

C 2021 International Union of Crystallography 